\title{
Feature Extraction from Terahertz Pulses for Classification of RNA data via Support Vector Machines
}

\author{
Xiaoxia Yin ${ }^{a}$, Brian W.-H. $\mathrm{Ng}^{a}$, Bernd Fischer, Bradley Ferguson ${ }^{a b}$, \\ Samuel P. Mickan ${ }^{a}$ and Derek Abbott $^{a}$ \\ ${ }^{a}$ Centre for Biomedical Engineering and School of Electrical \& Electronic Engineering, The \\ University of Adelaide, SA 5005, Australia; \\ ${ }^{b}$ Tenix - Electronic Systems Division 2nd Avenue, Technology Park, Mawson Lakes, SA 5095, \\ Australia
}

\begin{abstract}
This study investigates binary and multiple classes of classification via support vector machines (SVMs). A couple of groups of two dimensional features are extracted via frequency orientation components, which result in the effective classification of Terahertz (T-ray) pulses for discrimination of RNA data and various powder samples. For each classification task, a pair of extracted feature vectors from the terahertz signals corresponding to each class is viewed as two coordinates and plotted in the same coordinate system. The current classification method extracts specific features from the Fourier spectrum, without applying an extra feature extractor. This method shows that SVMs can employ conventional feature extraction methods for a T-ray classification task. Moreover, we discuss the challenges faced by this method. A pairwise classification method is applied for the multi-class classification of powder samples. Plots of learning vectors assist in understanding the classification task, which exhibit improved clustering, clear learning margins, and least support vectors. This paper highlights the ability to use a small number of features (2D features) for classification via analyzing the frequency spectrum, which greatly reduces the computation complexity in achieving the preferred classification performance.
\end{abstract}

Keywords: Terahertz, T-rays, support vector machines, pairwise classification, RNA

\section{INTRODUCTION}

T-rays have wavelengths in the submillimetre range from $30 \mu \mathrm{m}$ to $3000 \mu \mathrm{m}$, corresponding to the frequency range from $0.1 \mathrm{THz}$ to $10 \mathrm{THz}$ in the electromagnetic spectrum. T-rays have promising potential both in in vivo and in vitro biosensing ${ }^{1-3}$ owing to (i) their non-invasive property, and (ii) the fact that biomolecules ${ }^{4-6}$ have rich resonances in the $\mathrm{THz}$ region. The ultimate aim of our work is to perform automatic classification of data obtained from T-ray tomography. ${ }^{7}$ It is important to select effective feature extraction methods for different character representation of these signals. ${ }^{8}$ Signal processing methods are particularly proposed for the current experiment. In the current two experiments, input vectors are decomposed based on specific properties of their Fourier spectrum. ${ }^{9}$ As a result, a pair of specific features takes the place of a large number of measured features, in order to keep the number of features smaller than the number of observations to prevent over fitting. In this case, the over fitting problem is addressed with the added benefit of reduced computation complexity. Hence, the major issue for the current method is to select the specific feature for a given learning task.

The current experiments illustrate the potential of SVMs in pulsed signal recognition. The classification of two different types of RNA samples is of importance because it is a potential precursor for DNA sample classification in an in vivo environment. ${ }^{10}$ We also classify six different types of powder materials, motivated by security applications. We describe the methods for T-ray pulse classification. The input vectors are the measured raw T-ray signals, with the only preprocessing being a fast Fourier transform and deconvolution for Fourier spectrum analysis. Accordingly, an SVM kernel-based method can be directly applied to the specific features for linear mapping. The current experiments show that a Gaussian kernel and a polynomial kernel

Further author information: (Send correspondence to Xiaoxia Yin)

Xiaoxia Yin: E-mail: xxyin@eleceng.adelaide.edu.au, Telephone: 61-8-8303-6296

Micro- and Nanotechnology: Materials, Processes, Packaging, and Systems III, edited by Jung-Chih Chiao, Andrew S. Dzurak, Chennupati Jagadish, David Victor Thiel, Proc. of SPIE Vol. 6415, 641516, (2006) - 0277-786X/06/\$15 - doi: 10.1117/12.695629

Proc. of SPIE Vol. 6415 641516-1 
can work well, based on the conventional features. The main advantage of the current approach is that it is not necessary to carefully design a feature extraction mechanism, ${ }^{11}$ because the current feature extraction is straight forward for the training of the SVMs. Feature extraction and classification are performed with the aim of achieving high classification accuracy. A pairwise classification method ${ }^{12}$ is applied to multi-class recognition, with a basic scheme for binary classification. Gaussian and polynomial kernels are applied for two-class and multi-class classification, respectively. For the binary classification case, $2 / 3$ of total responses from each class are fed to the SVMs for training. Two hundred repeated random selections of training and testing sets from available inputs are taken to achieve the classification estimation. For multi-class classification, a leave-oneout method ${ }^{13,14}$ is used to design and estimate the classifier performance with pairwise classification. Visual classification performance is also shown for two dimensional features, which demonstrates the effective learning vector pattern and makes the design of SVMs understandable.

\section{OVERVIEW OF SVMS}

Support vector machines are binary classifiers, which classify data depending on a set of support vectors. ${ }^{15}$ The training data sets come from a set of labeled samples called learning vectors. We denote such a set of learning vectors as $\left(\mathbf{x}_{i}, y_{i}\right) \in R^{N} \times\{ \pm 1\}, i=1, \ldots, l$, and $y_{i}$ denote the output space corresponding to each input vector $\mathbf{x}_{i}$. The support vectors are subsets of the training data sets, which allow construction of a $l$ dimensional hyperplane in an $l$ dimensional feature space, as a boundary between the different classes. A decision function $f(\alpha): R^{N} \rightarrow \pm 1$ is achieved based on a given class function $f(\alpha): \alpha \epsilon \Lambda$, with the aim to correctly assign class labels to test samples $(\mathbf{x}, y)$. The Vapnik Chervonenkis (VC) dimension ${ }^{16-18}$ is a property of a set of functions $f(\alpha)$, which is defined as the maximum number of training points that can be segmented by $f(\alpha)$. Note that $\alpha$ corresponds to the weights and biases, which can be adjusted to label the output $f(\mathbf{x}, \alpha)$ based on the input $\mathbf{x}$. The expectation of the test error for a learning machine is:

$$
R(\alpha)=\int \frac{1}{2}|y-f(x, \alpha)| d P(\mathbf{x}, y)
$$

where, $R(\alpha)$ is called the expected risk. It is the quantity connected with density $p(\mathbf{x}, y)$ that we are ultimately interested in. The "empirical risk" $R_{\text {emp }}(\alpha)$ is defined to be the measured mean training error for a fixed, finite number of observations:

$$
R_{\mathrm{emp}}(\alpha)=\frac{1}{2 l} \sum_{i=1}^{l}\left|y_{i}-f\left(x_{i}, \alpha\right)\right| .
$$

The quantity $\frac{1}{2}\left|y_{i}-f\left(x_{i}, \alpha\right)\right|$, which is called the loss, takes on values equal to 0 or 1 . When we set the probability to $1-\eta$, we achieve the following bound:

$$
R(\alpha) \leq R_{\mathrm{emp}}(\alpha)+\sqrt{\left(\frac{h(\log (2 l / h)+1)-\log (\eta / 4)}{l}\right)} .
$$

The non-negative integer $h$ is called the VC dimension, and allows the measure of the capacity that is suitable for the amount of available training data. When a sufficiently small $\eta$ is selected, the right hand side of the equation is minimized, the $f(\mathbf{x}, \alpha)$ functions give the lowest upper bound of the actual risk. It is the basic idea of structural risk minimization.

Since minimizing the training error (the computation of VC-dimensions) does not guarantee a small test error, in order to make the decision function $f$ perform well on unseen pattern, the principle of structural risk minimization needs to be applied to minimize test error and achieve a capacity that is suitable for the amount of available training data sets.

The learning algorithm is designed to allow the support vectors computed via performing structure risk minimization. In other words, a VC-dimension bound is calculated to identify the optimal hyperplane and maximizes the margin of the nearest learning vectors. The decision hyperplane is calculated based on the following equation: 


$$
f(\mathbf{x})=\operatorname{sgn}\left(\sum_{i=1}^{s} y_{i} \alpha_{i} \mathbf{x}_{i} \cdot \mathbf{x}+b\right)
$$

where $\mathbf{x}_{i},(i=1 \ldots s)$ are support vectors, which are the closest points from the training vectors (learning vectors) to the separate hyperplane and sgn is called the signum function. The solution of this large-scale quadratic programming problem is applied to calculate the coefficients $\alpha_{i}$ and $b$. The procedure is to solve the dual problem, described as follows:

Maximize

$$
L(\alpha)=\sum_{i=1}^{l} \alpha_{i}-\frac{1}{2} \sum_{i, j=1}^{l} \alpha_{i} \alpha_{j} y_{i} y_{j}\left(\mathbf{x}_{i} \cdot \mathbf{x}_{j}\right)
$$

subject to $\sum_{i=1}^{l} \alpha_{i} y_{i}=0$ and $0 \leq \alpha_{i} \leq C$ for $i=1, \ldots, l$.

The penalty parameter $C$ is selected by the user, which is viewed as a regularization parameter for the linearly inseparable learning vectors aiming to accept the possible misclassifications.

SVMs use a kernel function, ${ }^{19,20}$ which allows fitting the hyperplane to the data. Instead of a linear classifier, which is applied to solve generic classification problems, the hyperplane ${ }^{21}$ needs to be augmented to fit the nonlinear decision surfaces. Dot product space, feature space $F$, is constructed by mapping data, which is realised by performing a nonlinear map $\phi: R^{N} \rightarrow F$. The above linear algorithm then can be applied in the feature space $F$. The solution satisfies the following function

$$
f(\mathbf{x})=\operatorname{sgn}\left(\sum_{i=1}^{s} y_{i} \alpha_{i} \phi\left(\mathbf{x}_{i}\right) \phi(\mathbf{x})+b\right) .
$$

This is a nonlinear function of the original input vectors $\mathbf{x}$.

According to Cover's ${ }^{22}$ theorem, a new feature space $F$ can be achieved via transforming a multidimensional space, where the dimensionality of the feature space are high enough to allow the target patterns linearly separable with a high probability. The inner products (dot products) enable the high dimensional space to be treated easily, which are indicated by the $\phi$ mapping. Accordingly, the kernel function $K$ is defined as:

$$
K(\mathbf{x}, \mathbf{y})=\phi(\mathbf{x}) \cdot \phi(\mathbf{y}) .
$$

The kernel production substitutes for all the occurrences of an dot product resulting from two mappings.

Table 1 summarizes the kernel functions for two common types of pattern classification: binary classification and multi-class classification realised via SVMs for the current experiments.

Table 1. Possible Kernel Functions and Types of Pattern Classification in the Current Experiments

\begin{tabular}{|c|c|}
\hline Inner product kernel & Type of pattern classification \\
\hline \hline Polynomial kernel: $K(\mathbf{x}, \mathbf{y})=(\mathbf{x} \cdot \mathbf{y})^{p}$ & Multi-class classification \\
\hline Gaussian kernel: $K(\mathbf{x}, \mathbf{y})=\exp \left(-\frac{1}{2 \sigma^{2}}\|x-y\|^{2}\right)$ & Binary classification \\
\hline
\end{tabular}

\section{TERAHERTZ PULSED CLASSIFICATION USING SUPPORT VECTOR MACHINES}

This section describes the classification system designed to assess the potential of SVMs in Terahertz (T-ray) pulsed classification. There are two target data sets, which need to be separated by SVMs: one is for RNA samples and the other is for powder samples. The former is to classify two classes of objects and the latter is to separate six various types of powdered materials. Gaussian kernels and polynomial kernels are applied for statistical feature extraction. Signal processing is applied to track the key features of training vectors for different classes of signals. 


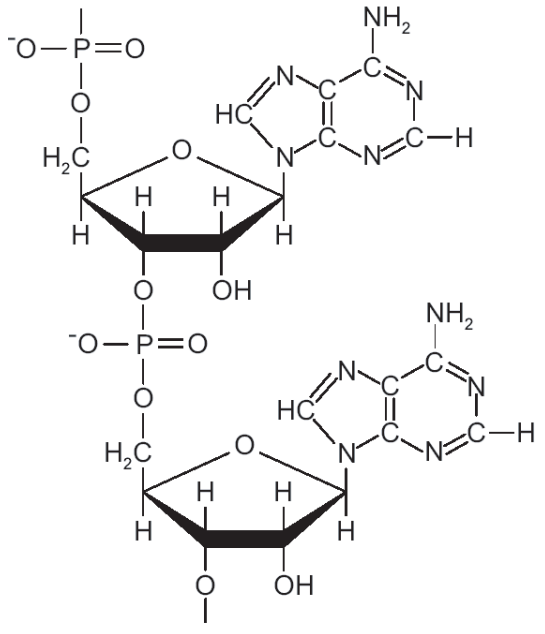

(a)

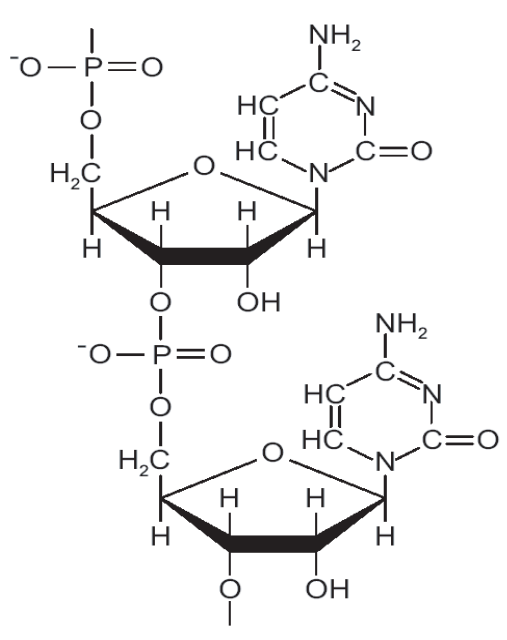

(b)

Figure 1. Chemical structure of (a) polyadenylic acid (poly-A) and (b) polycytidylic acid (poly-C). Potassium ions are applied to neutralize the negative charge of the backbone. After Fischer et. al. ${ }^{10}$

\subsection{RNA data representation and classification of two-class pulses}

Recently it was suggested that biological material can be detected by THz circular dichroism (TCD) spectroscopy, because many biomolecular crystals exhibit strong and specific absorption features in their dielectric spectra. ${ }^{10}$ Currently, the identification of the binding state of DNA is an especially interesting topic, which can be realised through applying $\mathrm{THz}$ techniques, i.e. loading a planar $\mathrm{THz}$ resonator with the sample material, in spite of the lack of characteristic absorption features in the $\mathrm{THz}$ region. In this experiment, we try to investigate the spectra in the frequency domain related to two different RNA polymer strands, polyadenylic acid (poly-A) and polycytidylic acid (poly-C) for the recognition task with potential to classify DNA.

Commercially available poly-A and poly-C potassium salts are used for the experiment (Sigma-Aldrich, product numbers P9403 and P4903). Fig. 1 illustrates the chemical structures of the polymers.

The RNA samples are prepared by the following steps: (i) The substrate is made of the commercially available cyclic olefin TOPAS (Greiner Bio-One) with transparent and dispersion-free properties in the THz frequency range; (ii) In order to achieve $\mathrm{THz}$ imaging, small liquid volumes are spotted in a $4 \times 4$ array of alternating poly-A and poly-C on a TOPAS substrate. Each spot with the diameter equal to 1 mm consists of $2 \mu \mathrm{l}$ of deionized water containing $0.2 \mathrm{mg}$ material.

As a result, the terahertz image is illustrated in Fig. 2, which is achieved by terahertz time-domain spectroscopy imaging system based on free-space propagation and aperture-less focusing of the THz beam. Each pixel in the image represents the normalized peak values corresponding to Poly-A and Poly-C. The sample consists of a $4 \times 4$ array of spots. We remove two of the spots from the substrate in order to identify the orientation of the substrate in the image. The spot of Poly-A is shown at the top left corner of the image, with weak transmission, compared to the spots of poly-C. The positions of poly-A and poly-C subimages are labeled in the diagram to the right of the picture.

Based on the positions of poly-A and poly-C at the terahertz image, we select 8-neighbor pixels around center position from each spot for the signal post-processing and classification. 


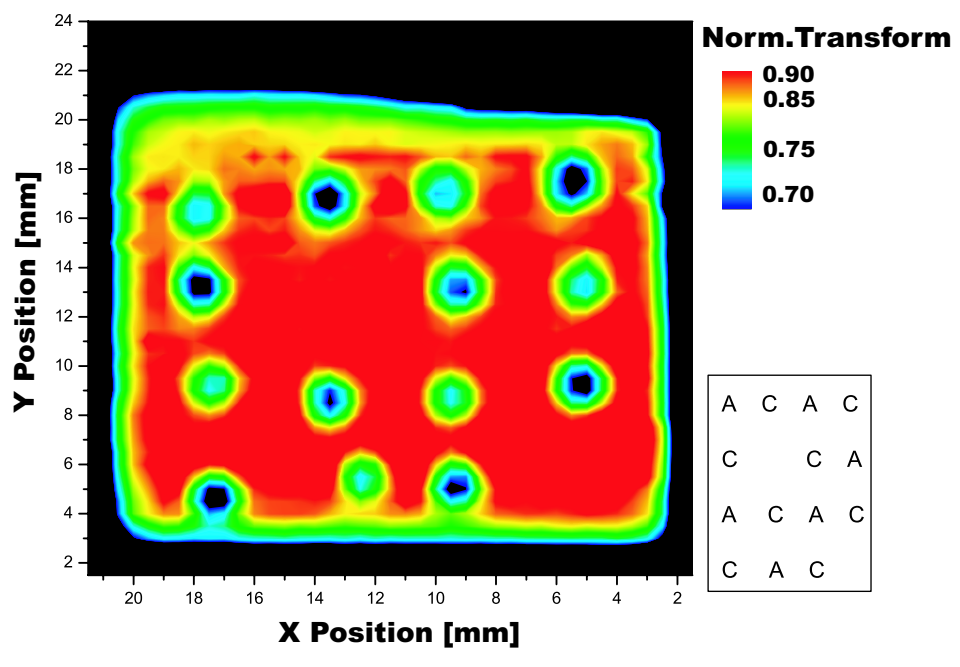

Figure 2. THz transmission image of the poly-A and poly-C, showing stronger absorption in poly-C compared to poly-A. Each spot contained $200 \mu \mathrm{g}$ of either poly-A or poly-C in alternating order, as indicated in the diagram to the right. The colour scale indicates the normalized peak values of the two RNA samples. After Fischer et. al. ${ }^{10}$

\subsubsection{The Fourier spectrum analysis for the classification of poly-A and ploy-C T-ray pulses}

The way to extract specific feature vectors in the frequency domain is realised by taking the Fourier transform after deconvolving measured signals with a reference pulse. ${ }^{23}$ As mentioned above, a pulse measured from the empty substrate is employed as reference and is deconvolved from the measured signals for the removal of the system response. ${ }^{23,24}$ The Fourier transform procedure achieves complex-valued spectra, phase and magnitude spectrum analysis, for finding orientation frequency components, the maximum magnitude, for the specific feature extraction of magnitude and phase from the poly-A and poly- $\mathrm{C}$ data.

The set of magnitude and phase features oriented by key frequency components constitutes a pair of feature subsets on which the classification is based. An important advantage of this approach is the small number of features (2D features), which allows SVMs to extract features directly from pulsed responses, hence the reduced computation expense with which classification can be achieved. The calculation complexity of standard quadratic programming is reduced ${ }^{25,26}$ from order $O\left(M^{3}\right)$ to order $O\left(M^{2}\right)$. Further, the sparse features help solve the over fitting problem. ${ }^{4,27}$ The input vectors occupy a size $M \times L$ matrix, where $M$ is equal to the number of input vectors (training vectors) and $L$ is the number of features of each input vector. If the limited measured dimensions of the training subsets $M$ is smaller than large scale of time series features $L$, it will cause difficulty in correctly assigning labels to target samples. Fast and sparse features overcome the computational disadvantages of SVMs.

Following the feature extraction, the kernel operation is performed on the calculated features in an SVM. The kernel first computes the inner product $\mathbf{z}=\mathbf{x} \cdot \mathbf{y}$, where $\mathbf{x}$ represents the input vectors and $\mathbf{y}$ indicates the calculated support vectors. A nonlinear transformation $\Theta$ is performed for mapping input pattern $\phi(\mathbf{x})$ to a linear space. In the case of a Gaussian kernel, this transformation is related to a Gaussian function $K(\mathbf{x}, \mathbf{y})=\exp \left(-\frac{1}{2 \sigma^{2}}\|x-y\|^{2}\right)$, where $\sigma$ is the Gaussian kernel width parameter. Accordingly, a SVM classifier needs to be applied to produce learning vector patterns in two dimension feature space. The scatter plot for learning vectors using a Gaussian kernel is illustrated in Fig. 3, which considers a two-class pulsed signal classification problem for the recognition of two types of RNA samples-poly-A and poly-C. It serves as a useful comparison with plotting machine learning realised via a linear kernel for classification tasks, illustrated in Section 3.2 on multi-class powder classification. The feature vectors consist of magnitude and phase, which are plotted in a two dimensional plane: $x$-axis is labeled by magnitude and $y$-axis labeled by phase. SVMs with the width parameter of Gaussian kernel $\sigma$ of 30 are trained by feeding 36 training vectors selected randomly from 48 patterns from each class. The orientation frequency is selected at the 19th frequency bin. The background colour shows the shape of the decision surface. Dark blue regions represent the class belonging to the poly-C sample labeled 


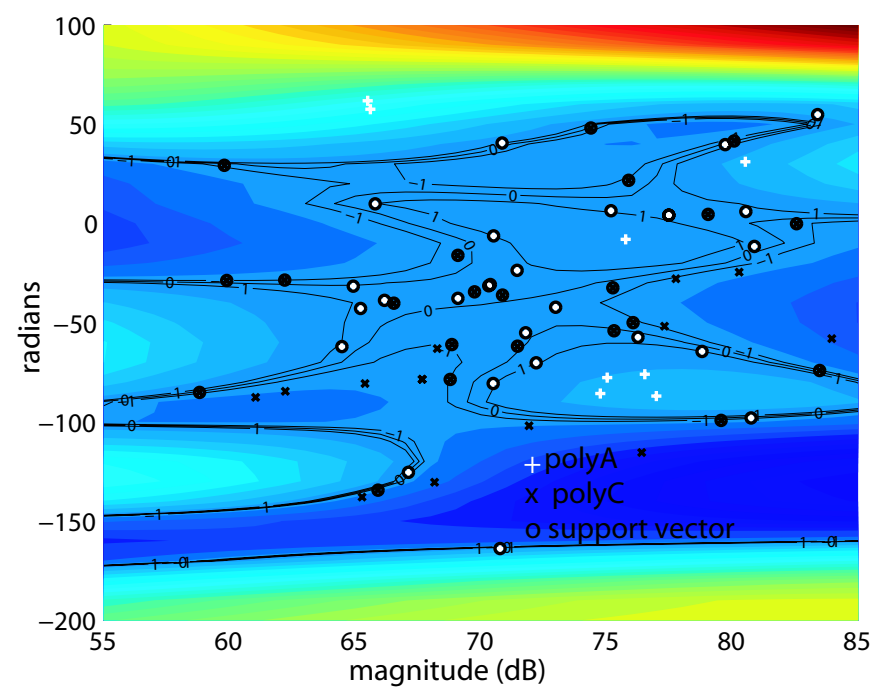

Figure 3. An illustration of binary classification for the recognition of RNA samples. A Gaussian kernel is used for mapping the training vectors to a $2 \mathrm{D}$ feature space. The penalty parameter $C$ is set to infinity and the width parameter of Gaussian kernel $\sigma$ equals 30. The background colour shows the contour shape of the decision surface. The learning vectors are approximately separated via applying Gaussian kernel for mapping.

by -1 ; and light blue regions indicate the class related to poly-A sample labeled by 1 . Separating hyperplanes for two classes are indicated by 0 . The circles represent the calculated support vectors. Compared to the training vectors, the number of support vectors are reduced, which takes on an important role in achieving the ideal shape of hyperplanes and facilitating computation of the classification algorithm. In this case, the machine learning for two-class samples - poly-A and poly-C denoted by white "+" and black " $\times$ " are approximately separate by their own boundary lines though there is a little overlapping.

The parameters that need to be tuned in the SVMs include the kernel parameters and penalty parameter $C$. The use of validation data can be viewed as a straightforward method for tuning. This method is to select validation subsets from training data sets and put aside the remaining. ${ }^{11}$ However, it is proposed that for the current experiment, due to the the size of training data sets, is quite limited, it is better to find an alternative way to tune the necessary parameter. In this paper, the proposed method for tuning the parameters is based on the test error, because we are more concerned with minimizing the test error rather than minimizing the training error. The corresponding training vectors plotted in the two dimensional feature space, illustrated in Fig. 3, indicates that in this case, the two object classes can be recognized in obvious boundary regions belonging to their own classes.

Fig. 4(a) displays two obvious separate phase curves for two classes of spectra-poly-A and poly-C, which are obtained by Fast Fourier Transform (FFT) of the T-ray pulses with linear extrapolation. Fig. 4(b) is the plot of magnitude versus different frequency steps with cutoff frequency at the 160th frequency bin. It is observed that the magnitude at frequency bin 19 reaches maximum value. We view frequency bin 19 as a key frequency and calculate the corresponding phase and magnitude, both of which are as a pair of extracted features and input to SVMs. The Gaussian kernel as a channel filter realises the final feature mapping from a non-linear feature space to a linear one. Accordingly, we apply a SVM classifier to produce a learning vector pattern in the two dimensional feature space - magnitude and phase as a pair of coordinates. We obtain acceptable classification accuracy after 200 random selections of training vectors, fed to the SVMs. The detailed experimental results are analyzed in the next section. 


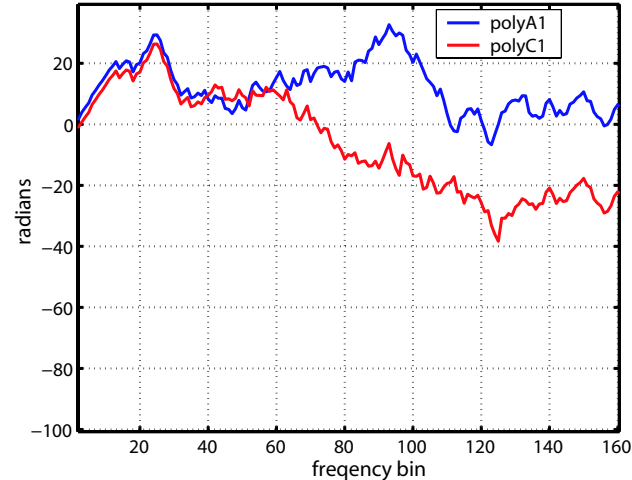

(a)

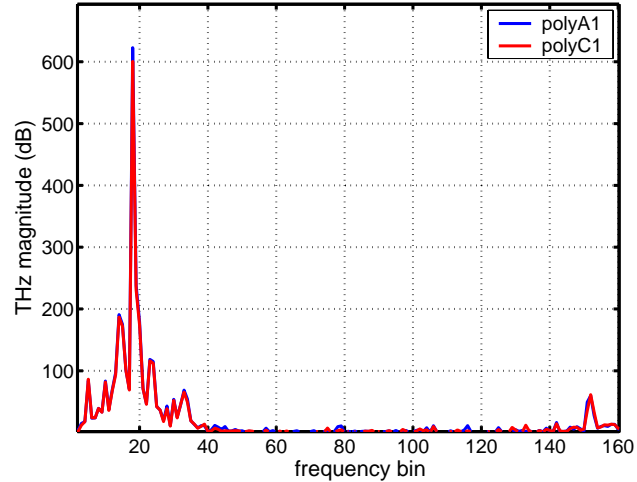

(b)

Figure 4. Illustration of T-ray spectra of RNA. (a) Displays two obvious separate phase curves for two classes of spectrapoly-A and poly-C, which are obtained by down sampling the Fast Fourier Transform (FFT) of T-ray pulses with linear extrapolation. (b) The plot of magnitude versus different frequency bins with largest magnitude at frequency bin 19.

\subsection{Pairwise classification of T-ray pulses for different types of powder}

The previous section described an SVM for two-class pulsed signal classification, which is also called a dichotomy. This is appropriate for the object detection application of two-class T-ray pulses and discriminating an object from the background. However, the majority of object recognition problems consist of more than two substances. Consequently, extended SVMs are required for application to multi-class pulsed signals. The optimal design of multi-class SVM classifier is an area of active research. One frequently used method is a pairwise classifier, based on one-against-one decomposition, ${ }^{12}$ which works by using a decision function $f_{k l}$. Here, $k l$ indicates each pair of classes selected from separated target classes. Since the symmetric property of pairwise approach, it holds $f_{k l}=-f_{k l}$, where $f_{k l}$ satisfies the following equation:

$$
f_{k l}(\mathbf{x})=\mathbf{w}_{k l} \mathbf{x}+b_{k l}
$$

where $\mathbf{w}$ is viewed normal to the hyperplane between class $k$ and class $l,|b| /\|\mathbf{w}\|$ is the perpendicular distance from the hyperplane to the origin, and $\|\mathbf{w}\|$ is the Euclidean normal of $\mathbf{w}$ with vector dimension of $M$.

The signum function is used for the hard threshold decisions:

$$
\operatorname{sgn}\left(f_{k l}(\mathbf{x})\right)=\left\{\begin{array}{l}
1, f_{k l}(\mathbf{x})>0 \\
-1, f_{k l}(\mathbf{x}) \leq 0
\end{array}\right.
$$

where $n$ is the number of the separated target classes, and the class decision can be achieved by summing up the according pairwise decision functions:

$$
f_{k}(\mathbf{x})=\sum_{k \neq l, l=1}^{n} \operatorname{sgn}\left(f_{k l}(\mathbf{x})\right) .
$$

Assign a label to the class: $\arg \max f_{k}(\mathbf{x}),(k=1, \ldots, n)$. The max number of votes for $k$ classes holds $\max _{k} f_{k}=(k-1)$. If Eq. 10 is satisfied $\max _{k} f_{k}<(k-1), \mathbf{x}$ is unclassifiable. The pairwise classification converts the $n$-class classification problem into $n(n-1) / 2$ two-class problems which cover all pairs of classes.

The procedure for pairwise classification is illustrated in Fig. 5. 


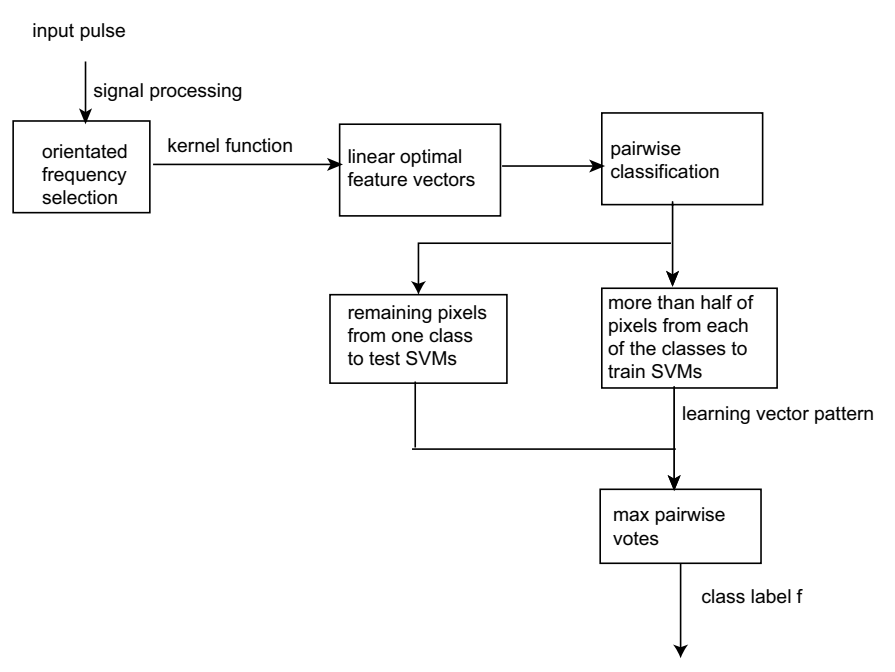

Figure 5. Illustration of the procedure for pairwise classification.

\subsubsection{Representation of powder samples}

Recently, classifying powdered substances has become attractive research topic with applications in substance detection for security. A general question is ti explore the ability of $\mathrm{THz}$ spectroscopy to detect different densities, thicknesses and concentrations of specific powders. To investigate this problem, we conduct a preliminary exploration of different powder recognition tasks with $2 \mathrm{~mm}$ thickness for six different powdered substances and their holder. They are: sand, talcum, salt, powdered sugar, wheat flour, and baking soda.

The sample holder is shown in Fig. 6 and has an ability to accurately control the thickness of the powders. There are two teflon blocks, which can be separated under control. They are mounted on a manual translation stage to provide the required gap of $2 \mathrm{~mm}$, where a plastic bag containing the powdered substances can be inserted between the teflon blocks. This procedure guarantees a relatively consistent powder density and accurate control over the powder thickness of $2 \mathrm{~mm}$.

A traditional T-ray imaging system is used to detect T-ray responses based on the THz-TDS technique. There is an X-Y translation stage involved for mounting the teflon sample holder and fixing its position in the T-ray beam. At THz frequencies, teflon is dispersionless. As teflon has a very low absorption coefficient, there is minimal distortion while the $\mathrm{THz}$ pulse propagates through the holder. A $2 \mathrm{D} \mathrm{THz}$ image of the sample can be obtained after inserting a powder sample. This image allows the effects of different scattering paths and minor variations in powder thickness and density to be observed. In general a $1 \mathrm{D}$ image is sufficient, and 50 pixel images (with a pixel spacing of $100 \mu \mathrm{m}$ ) can be acquired in under 30 minutes.

\subsubsection{Fourier spectrum analysis for multi-class classification}

As mentioned above, the detection procedure works on a modified transmitted time-series $\mathrm{THz}$ pulsed responses. An FFT and a de-convolution are taken between the holder (reference pulse) and the T-ray pulses of powder samples for isolation of system responses and for analysis of Fourier coefficients. Fig. 7 shows the phase and magnitude plots in the frequency domain from one pixel of salt image data, with a cutoff frequency equal to 4 THz. It is obvious that the subimage at the bottom of Fig. 7(a) shows a sharp change of magnitude at the second frequency bin. Accordingly, with the number of training subsets of 49, we plot the phase and magnitude based on two classes learning vectors and multi-classed learning vectors, by applying the pair Fourier features at the second frequency bin, then we produced the learning vector pattern for two-classes and multi-class recognition, shown in Fig. 7(b) and Fig. 8, respectively. For powder classification, a polynomial kernel is employed for optimal classification performance. The two-class powder samples, sand and salt samples, are linearly separable, with circles describing the calculated support vectors, which decide the linear optimal hyperplane between two classes. The solid lines above and below the hyperplane depict the \pm 1 range along the separating surface. Compared to 


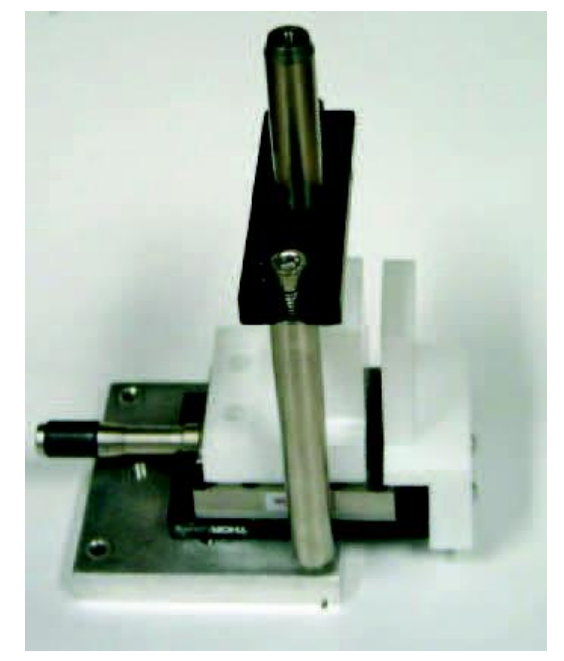

Figure 6. Photo of a teflon sample holder for measurement fixed thickness of powdered samples. One of the teflon blocks is fixed, while the position of the other is controlled using the manual translation stage. The gap between the two blocks may be adjusted to allow $2 \mathrm{~mm}$ thicknesses of powder to be considered.

the training vectors, the number of support vectors are greatly reduced, which allows easy classification task. In two-class classification, the penalty parameter $C$ equals to infinite and the polynomial kernel degree equals 1.

For the multi-class case, a polynomial kernel with degree of 1 is applied for linear mapping, with a truncated terahertz frequency of $3 \mathrm{THz}$. The penalty parameter $C$ is set to 1000. The relevant decision functions for the pairwise approach are shown in Fig. 8 with the number for the recognition of the various decision surfaces corresponding to the different pairwise classes. The summing up of the pairwise votes yields the borders easily. The small red region at the left hand outlined by border line 5 is an undecided class region. This is because the maximum number of votes (top scores) in the region are smaller than $(k-1)=5$. The six classes are well clustered and therefore perfectly separated. The undecided class region has no relevance for the class decision. It is obvious that the number of the support vectors for the single decision is quite smaller, which results in a fast adaption and better boundary shape though the computation is increased by a factor of $(k-1) / 2$. The support vectors as the "most important" data points are identified with extra circles.

\section{EXPERIMENTAL RESULTS}

To verify the effectiveness of the proposed method, experiments were performed on T-ray pulsed data related to RNA samples and several types of powder substances. The T-ray pulses are obtained by transmitted terahertz time domain spectroscopy imaging system, with an advantage of capturing both phase and amplitude information, without resort to the Kramers-Kronig ${ }^{28}$ relation. The current T-ray imaging system supplies three dimensional data information for each T-ray image.

\subsection{RNA classification via Fourier coefficient spectrum}

RNA image data occupies a size equal to $60 \times 50=3000$ pixels. As for each pixel, the number of time samples is 350 , which was accordingly truncated at 175th frequency bin corresponding to frequency of $4 \mathrm{THz}$ because of the symmetry of spectrum. The 3000 image data set consists of background data information - a TOPAS substrate image and target object data sets - poly-A and poly-C image data. The population of the possible applied data set for poly-A and poly-C equals 48, respectively. In order to obtain reduced dimensions of feature subsets and make them discriminable for each class, the values in relation to magnitude and phase are calculated, and a pair of feature vectors: magnitude versus phase are extracted based the orientation frequency that corresponds to the maximum magnitude. It follows a deconvolution procedure for removing the system response. As for reference pulse, we choose the pulse from a pixel of substrate image data. The pixels lying on the boundaries of each class 

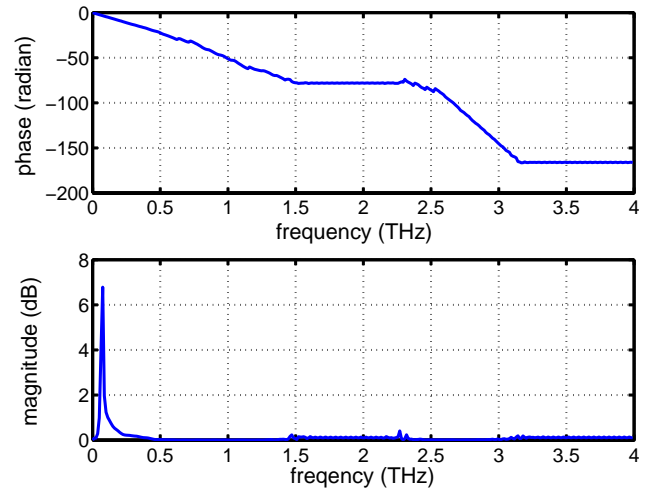

(a)

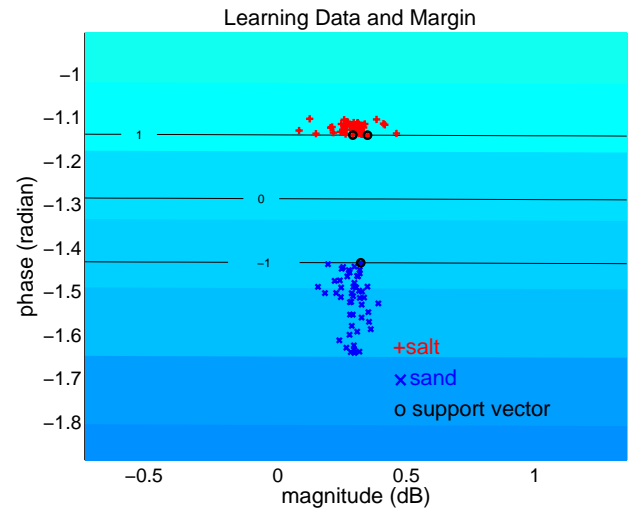

(b)

Figure 7. (a) Illustration of Fourier spectrum. The top subimage shows phase versus terahertz frequency and the bottom subimage shows magnitude versus terahertz frequency. (b) Plot of learning vector pattern with 49 pixels from two-class powder samples, sand and salt samples, with phase and amplitude as a pair of coordinates. Polynomial kernel with degree of 1 is applied for linear mapping. The penalty parameter $C$ is set to infinity.

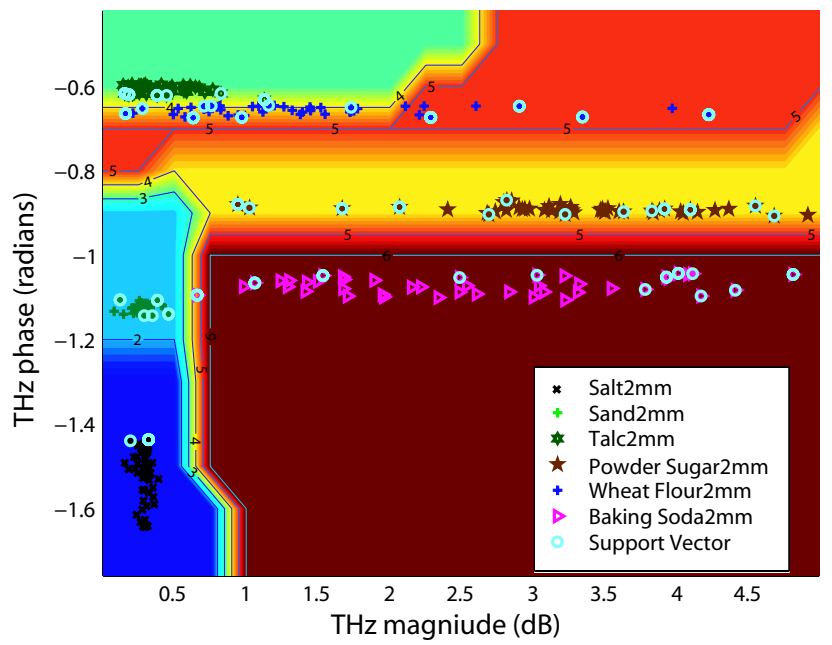

Figure 8. Learning vectors for the six-class examples are plotted for the illustration of the linear decision function between the pairs of classes by applying a polynomial kernel for mapping with degree of 1 . There are 49 pixels selected randomly from each of six classes of powder samples. The small red region on the left hand side remains undecided.

are excluded from training and test vectors due to the difficulties involved in manually assigning the desired values for pattern recognition.

Gaussian kernels are applied because of the preferred classification performance and fit the RNA data well. All the classification runs are performed using a $2.4 \mathrm{GHz}$ Pentium4 CPU. The average time spent classifying the two-classes RNA samples is 2.74 seconds after 18 runs of measurements.

To evaluate the effect of the Gaussian kernel for the RNA sample classification, suitable values of $C$ and $\sigma$ are evaluated via parametric search. The error rate, the number of support vectors and the elapsed time are compared. The number of training vectors is $2 / 3$ of the total size of input vectors, which are selected randomly from 48 image data of each class. The remaining pixels are used for testing the SVMs. In order to acquire 
effective classification, the repeated selection of test and training vectors are conducted, and the number of repeated selection approaches 200 . The preferred classification performance occurred at the penalty parameter $C=0.4$ and $\sigma=7$, with classification accuracy of $71 \%$. For the single calculation, there exists 21 classified test vectors out of 24 test vectors, with classification accuracy reaching $88 \%$.

The feature extraction method realised by orientation frequency components is particularly attractive when the input vectors come from large data sets. However, it should be noted that the current resultant classification of RNA samples is limited by laser fluctuation occurring in the measurement procedure between an RNA sample and the substrate reference. In addition, the RNA data is measured on a very thin layer of the substrate (around $40 \mu \mathrm{m})$. The propagation delay is often less than 1 sampling period, which makes model fitting difficult.

The number of the computed support vectors is small compared to the number of training vectors. The relatively small number of SVs reveals that the machine learning task is low in complexity.

\subsection{Multi-class powder classification via Fourier coefficient spectrum}

Table 2 and Table 3 illustrate multi-class classification performance via applying a pairwise classification method with the application of a polynomial kernel on Fourier spectral features. Elapsed time and the number of SVs are also illustrated in the two tables. Leave-one-out estimator is used for the design and analysis of SVMs. The total size of analyzed vectors of each target class is $50 \times 400=20000$ before feature extraction. For training, $49 \times 6=294$ pixels from all the classes are input to SVMs. The remaining 1 pixel from each class is used to test the SVMs. The SVM experiments are repeated $6 \times 5=30$ runs. The maximal vote is selected as a winner for the recognition of a target. The averaged classification accuracy in relation to each powder sample is shown in the two tables based on the various penalty parameter $C$ and the degree $p$ of a polynomial kernels. The best classification accuracy is achieved when the penalty parameter $C=100$ and the degree of a polynomial kernel $p=3$, with the accuracy being universally $100 \%$ except for $98 \%$ classification rate achieved for the recognition of flour sample. It should be noted that the relatively few SVs, with a average number of 55 , are detected when compared to the large number of input training vectors, with the number of $50 \times 6=300$, which allows moderate size quadratic programming. The average elapsed time is measured for each class classification, which is approximately equal to 9 seconds when using a polynomial kernel with degree from 1 to 3 .

Table 2 shows that the classification accuracy versus the penalty parameter $C$ of 10,100 and 1000 with a fixed degree of a polynomial kernel equal to 2. It is obvious that, with the increase of $C$, there exists improved classification accuracy, reduced the averaged number of support vectors and the elapsed time. When $C$ reaches 100 , a saturation point is reached.

Table 3 shows that the classification accuracy versus the polynomial kernel of 1,2 and 3 and a fixed penalty parameter $C$ of 100 . It is also clear that, with the increase of $p$, there exist improved classification accuracy, reduced the averaged number of support vectors and the elapsed time. When $p$ reaches 3 , a saturation point is reached. It should be noted that in the following two tables, the $97.96 \%$ and $93.8 \%$ classification accuracy correspond to the number of the correct classified test vectors equal to 48 and 46 with 1 and 3 test errors, respectively.

Table 2. Classification rates (\%), number of SVs and elapsed time are illustrated for RNA classification using varying penalty parameter $C$ and the polynomial kernel $p$ of 3 . The Fourier spectral features were extracted. Here, $97.96 \%$ and $93.8 \%$ classification accuracy correspond to the number of the correct classified test vectors equal to 48 and 46 with 1 and 3 test errors, respectively.

\begin{tabular}{|c|c|c|c|c|c|c|c|c|}
\hline \multirow{2}{*}{$C$} & \multicolumn{6}{|c|}{ each class name and classification accuracy (\%) } & $\sharp$ of SVs & elapsed time $(s)$ \\
\cline { 2 - 9 } & salt & sand & talcum & sugar & flour & soda & & \\
\hline 10 & 100 & 100 & 97.96 & 100 & 93.88 & 97.96 & 79 & 58.6720 \\
\hline 100 & 100 & 100 & 97.96 & 100 & 100 & 97.96 & 60 & 55.3130 \\
\hline 1000 & 100 & 100 & 97.96 & 100 & 100 & 97.96 & 50 & 50.8120 \\
\hline
\end{tabular}


Table 3. Classification accuracy (\%), the number of SVs and the elapsed time for RNA classification using varying polynomial kernel degree $p$ and fixed penalty parameter $C$ of 100 . The Fourier coefficients are the extracted features. Here, $97.96 \%$ and $93.8 \%$ classification accuracy correspond to the number of the correct classified test vectors equal to 48 and 46 with 1 and 3 test errors, respectively.

\begin{tabular}{|c|c|c|c|c|c|c|c|c|}
\hline \multirow{2}{*}{$p$} & \multicolumn{6}{|c|}{ each class name and classification accuracy (\%) } & $\sharp$ of SVs & elapsed time $(s)$ \\
\cline { 2 - 9 } & salt & sand & talcum & sugar & flour & soda & & \\
\hline 1 & 100 & 100 & 97.96 & 100 & 100 & 97.96 & 64 & 54.4690 \\
\hline 2 & 100 & 100 & 97.96 & 100 & 100 & 97.96 & 60 & 55.3130 \\
\hline 3 & 100 & 100 & 100 & 100 & 97.96 & 100 & 41 & 49.2820 \\
\hline
\end{tabular}

\section{CONCLUSION}

This paper describes an SVM-based T-ray pulsed signal classification method to estimate the potential of SVMs in the classification of RNA samples, poly-A and poly-C, and the classification of six types of powder materials. Pairwise classification is employed for the multi-class powder samples. The suggested method is to put the original T-ray pulses into SVMs, which does not involve any external feature extraction scheme except for the adoption of the normalization and fast Fourier transform for signal and spectrum analysis. The principle for this ability is that SVMs allow processing the sparse features for machine learning in low-dimensional feature spaces. The validity of using Gaussian and polynomial kernels is supported by effective classification performance of the above two feature extraction procedures.

Future studies will investigate further signal processing techniques and statistical modeling ${ }^{29,30}$ based methods for different feature extraction of T-ray pulses, with a link to SVMs. Autoregressive modeling ${ }^{31}$ can be employed as a technique to achieve the decomposition of large number of measured time samples. Absorption coefficients and refractive index are also suggested to be used as good configuration for the key features extraction, especially while analyzing the RNA spectrum data affected by obvious etalon artifacts. Meanwhile, more experiments related to DNA need to be carried out for the exploration of different feature configuration and different multiclass classification methods.

\section{REFERENCES}

1. R. M. Woodward, B. Cole, V. P. Walace, D. D. Arnone, R. Pye, E. H. Linfield, and M. Pepper, "Terahertz pulse imaging in reflection geometry of human skin cancer and skin tissue," Journal of Investigative Dermatology 120, pp. 3853-3863, 2002.

2. D. Mittleman, R. Neelamani, R. B. J. Rudd, and M. Koch, "Recent advances in terahertz imaging," Appl. Phys. B. 68, pp. 1085-1094, 1999.

3. D. Mittleman, R. Jacobsen, and M. Nuss, "T-ray imaging," IEEE J. Sel. Topics Quantum Electronics 2(679), 1996.

4. B. Withayachumnankul, B. Ferguson, T. Rainsford, D. Findlay, S. P. Mickan, and D. Abbott, "T-ray relevant frequencies for osteosarcoma classification," in Proceedings of SPIE Photonics: Design, Technology, and Packaging II, 6038, pp. 1H1-1H14, (Brisbane, Australia), 2005.

5. B. Ferguson, S. Wang, D. Gray, D. Abbott, and X. C. Zhang, "Identification of biological tissue using chirped probe THz imaging," Microelectronics Journal 33(12), pp. 1043-1051, 2002.

6. H. Siegel, "Terahertz technology in biology and medicine," IEEE Transactions on Microwave Theory and Techniques 52(10), pp. 2438-2447, 2004.

7. X. Yin, B. W.-H. Ng, B. Ferguson, S. P. Mickan, and D. Abbott, "Information fusion and wavelet based segment detection with applications to the identification of 3D target T-ray CT imaging," IEEE Sensor Letters, 2006, accepted.

8. Ø. D. Trier and T. M. Jain, A. K. Taxt, "Feature extraction methods for character recognition - a survey," Pattern Recognition 29, pp. 641-662, 1996.

9. S. Wang, B. Ferguson, and X.-C. Zhang, "Pulsed terahertz tomography," Journal of Physics D: Applied Physics 37, pp. R1-R36, (See also Erratum: Journal of Physics D : Applied Physics, 37, p. 964, 2004), 2004. 
10. B. Fischer, M. Hoffmann, H. Helm, R. Wilk, F. Rutz, T. Kleine-Ostmann, M. Koch, and P. Jepsen, "Terahertz time-domain spectroscopy and imaging of artificial RNA," Opt. Express 13, pp. 5205-5215, 2005.

11. K. I. Kim, K. Jung, S. H. Park, and H. J. Kim, "Support vector machines for texture classification," IEEE Transactions on Pattern Analysis and Machine Intelligence 24(11), pp. 1542 - 1550, 2002.

12. B. Schölkopf, C. J. C. Burges, and A. J. Smola, Advances in Kernel Methods: Support Vector Learning, The MIT Press, Cambridge, MA, USA, 1998.

13. K. Fukunaga and D. M. Hummels, "Leave-one-out procedures for nonparametric error estimates," IEEE Transactions on Pattern Analysis and Machine Intelligence 11(4), pp. 421-423, 1989.

14. K. Fukunaga and D. L. Kessell, "Nonparametric bayes error estimation using unclassified samples," IEEE Transactions on Information Theory IT-19(4), pp. 434-440, 1973.

15. N. Cristianini and J. Shawe-Taylor, An Introduction to Support Vector Machines and Other Kernel Based Methods, Cambridge University Press, Cambridge, UK, 2000.

16. V. N. Vapnik, Statistical Learning Theory, John Wiley \& Sons, Inc., Wiley, New York, 1998.

17. V. N. Vapnik, The Nature of Statistical Learning Theory, Springer-Verlag, New York, 1995.

18. K.-R. Muller, S. Mika, G. Ratsch, K. Tsuda, and B. Schölkopf, "An introduction to kernel-based learning algorithms," IEEE Transactions on Neural Networks 12(2), pp. 181-201, 2001.

19. B. Schölkopf and A. Smola, Learning with Kernels Support Vector Machines, Regularization, Optimization, and Beyond, MIT Press, Cambridge, MA, 2002.

20. J. Shawe-Taylor and N. Cristialnini, Kernel Methods for Pattern Analysis, Cambridge University Press, Cambridge, UK, 2004.

21. M. A. Hearst, "Trends controversies: Support vector machines," IEEE Intelligent Systems 13(4), pp. 18-28, 1998.

22. T. M. Cover, "Geometrical and statistical properties of systems of linear inequalities with applications in pattern recognition," IEEE Transactions on Electronic Computers 14, pp. 326-334, 1965.

23. B. Ferguson and D. Abbott, "Denoising techniques for terahertz responses of biological samples," Microelectron. J. 32(12), pp. 943-953, 2001.

24. L. Duvillaret, F. Garet, and L. Coutaz, "A reliable method for extraction of material parameters in terahertz time-domain spectroscopy," IEEE Journal of Selected Topics in Quantum Electronics 2(3), pp. 739-746, 1996.

25. J. C. Platt, Advances in Kernel Methods: Support Vector Learning, pp. 185-208, MIT Press, Cambridge, MA, 1999.

26. H. J. Shin and S. Cho, "Fast pattern selection for support vector classifiers," in Proc. of the 7th PacificAsia Conference on Knowledge Discovery and Data Mining (PAKDD), pp. 1008-1015, (Springer-Verlag, Heidelberg), 2003.

27. I. Guyon, J. Weston, and S. Barnhill, "Gene selection for cancer classification using support vector machines," Machine Learning 46, pp. 389-422, 2002.

28. K. Waters, B. Hoffmeister, and J. Javarone, "Application of the kramers-kronig relations to measurements of attenuation and dispersion in cancellous bone," in Proc. of 2004 IEEE: Ultrasonics Symposium, 1, pp. 561-564, (Montreal, Canada), 2004.

29. J. Schürmann, Pattern Classification: A Unified View of Statistical and Neural Approaches, John Wiley and Sons, Inc., New York, 1996.

30. R. Schalkoff, Pattern Classification: Statistical, Structural and Neural Approaches, John Wiley and Sons, Inc., New York, 1992.

31. C. Therrien and A. Oppenheim, Discrete Random Signals and Statistical Signal Processing, Prentice Hall, New Jersey, USA, 1992. 